\title{
Review: evidence is lacking that adults given fluids 1.5 to 3 hours preoperatively have greater risks of aspiration or regurgitation than those given a standard fast
}

Brady M, Kinn S, Stuart P. Preoperative fasting for adults to prevent perioperative complications. Cochrane Database Syst Rev 2003;(4):CD004423 (latest version 27 Aug 2003).

\section{$Q$ In adults, what are the effects of different preoperative fasting regimens (duration, type, and volume of permitted intake) on perioperative complications and patient wellbeing?}

METHODS

Data sources: Medline (1966 to August 2003), CINAHL (1982
to August 2003), the Cochrane Central Register of Controlled
Trials (second quarter, 2003), and the UK National Research
Register (August 2003); bibliographies of relevant studies and
conference proceedings; and experts.
Study selection and assessment: randomised controlled trials
(RCTs) or quasi-RCTs that compared the effects of different
preoperative fasting regimens in terms of duration of fast, type of
permitted intake, and volume of permitted intake before general
anaesthesia; included adults श18 years of age; and reported
postoperative complications. Studies of healthy patients having
general anaesthesia or those at high risk of regurgitation or
aspiration during anaesthesia (ie, patients who were pregnant,
postpartum, obese, elderly, or had gastric disorders) were
included. A standard fast was defined as nil by mouth from
midnight before morning surgery and a small breakfast early in
the morning before afternoon surgery. Intake of a small amount
of fluid (10-30 ml) to assist intake of oral medication was
permitted. Studies were assessed for methodologic quality
(randomisation method, allocation concealment, blinding, a
priori sample size calculation, and whether analysis was by
intention to treat).
Outcomes: perioperative complications including aspiration;
regurgitation; related morbidity (eg, aspiration pneumonia) or
death; volume, pH, or volume and pH of gastric contents; thirst;
hunger; pain; nausea; vomiting; and anxiety.

\section{MAIN RESULTS}

22 trials ( 38 randomised controlled comparisons, $\mathrm{n}=2270$ ) met the selection criteria. 15 trials reported patients' experience of the fasting process (ie, thirst, hunger, pain, nausea, vomiting, and anxiety). Most trials focused on generally healthy patients not thought to be at increased risk of regurgitation or aspiration during anaesthesia, although 3 trials included patients considered to be at a higher risk (obese patients in 1 trial and postpartum women in 2 trials). Trials primarily examined patients having elective surgery.

Few trials reported outcomes of aspiration, regurgitation, or related morbidity; intraoperative gastric volume and $\mathrm{pH}$ were more common outcomes. 6 trials compared a shortened fluid fast (fluids given 1.5 to $3 \mathrm{~h}$ preoperatively) with a standard fast, and none reported any aspiration or regurgitation. 24 comparisons examined a shortened fluid fast $v$ a standard fast and found no differences in volume or $\mathrm{pH}$ of gastric contents. Fluids permitted preoperatively included water, coffee, fruit juice, clear fluids, and other drinks. In 9 comparisons, patients given a drink of water preoperatively $(n=319)$ had a lower volume of intraoperative gastric contents than those who had a

For correspondence: $\operatorname{Dr} M$ Brady, Nursing Research Initiative for Scotland, Glasgow, UK. m.brady@gcal.ac.uk

Source of funding: Chief Scientist Office, Scottish Executive Health Department, UK. standard fast $(\mathrm{n}=292)$ (weighted mean difference $-6.16 \mathrm{ml}, 95 \% \mathrm{CI}$ -9.41 to -2.91$)$. 24 comparisons examined different volumes of preoperative intake (ie, low, medium, high, or unlimited) versus a standard fast and found no differences in perioperative outcomes.

\section{CONCLUSION}

Evidence is lacking that adults given fluids 1.5 to 3 hours preoperatively have a greater risk of aspiration or regurgitation than those who have a standard fast.

\section{Commentary}

B rady et al are to be commended on their comprehensive review of preoperative fasting to prevent perioperative complications because research on this subject poses several challenges. Most of the identified RCTs had small sample sizes (usually $<100$ patients per trial), and the methodological quality of some trials was inadequate. The authors' primary objectives were to systematically identify, appraise, and synthesise reliable evidence from research on different preoperative fasting regimens (length of the fast and the form and volume of intake allowed) in terms of patient safety and comfort in different adult populations. They met their objective of clarifying the evidence related to the different preoperative fasting regimens and their effects on patient comfort (eg, thirst). However, Brady et al were unable to synthesise the evidence from different adult populations because most studies focused on healthy or non-pregnant adults, or adults having elective surgery. Most studies reported outcomes of gastric content volume and $\mathrm{pH}$ rather than more clinically significant outcomes, such as perioperative morbidity, in assessing various preoperative fasting regimens. Brady ef al declared that the critical values at which patients have an increased risk of aspiration related complications are unclear and have not been validated. They also note that it would be unethical to conduct a trial that could place patients at risk of aspiration.

This review will be helpful to nurses in preoperative and operating room settings because it supports existing anaesthesiology guidelines. ${ }^{2} 2$ The review relates to the use of clear fluids up to 1.5 hours preoperatively for healthy adults having elective surgical procedures. However, this patient group is not considered to be at a higher risk of the perioperative complications of regurgitation and aspiration. The authors noted that few studies focus on at risk populations. This limits their ability to make recommendations for at risk groups, including patients with obstetric conditions, diabetes or morbid obesity, gastro-oesophageal reflux, and those having emergency surgery who have not had a preoperative fast. Brady et al note that future studies need to examine the preoperative fasting needs of these patient groups that are of concern.

Hilda Power RN, MN, CPN(C) IWK Health Centre Halifax, Nova Scotia, Canada

1 American Society of Anesthesiologists. Practice guidelines for preoperative fasting and the use of pharmacologic agents to reduce the risk of pulmonary aspiration: application to healthy patients undergoing elective procedures. Anesthesiology 1999:90:896-905.

2 Canadian Anesthesiologists' Society. Guidelines to the practice of anesthesia. Revised edition, 2003 (accessed 13 February 2004) http:// www.cas.ca/members/sign_in/guidelines/practice_of_anesthesia/ 